\title{
ON THE RING OF QUOTIENTS OF A NOETHERIAN RING
}

\author{
J. Lambek
}

(received August 17, 1964)

This paper is largely an expository account of known facts, but it contains at least one result believed to be new, Proposition 6 .

Our main technique is the method of lifting idempotents developed in Part I. This has been treated in the literature, but not quite in the generality required here. It turns out that much of classical artinian ring theory can be done for the semi-perfect rings introduced by Bass, as will have been noticed by many other people.

In Part II we consider Johnson's extended centralizer and Utumi's maximal ring of right quotients of a right noetherian ring. The former is semi-perfect and the latter is almost as nice.

Finally I have yielded to the temptation to apply these results to prime rings. While this is old hat, I have included a proof of the crucial lemma for Goldie's Theorem which appears to be shorter than any in the literature.

Throughout this paper rings are assumed to be as sociative with unity element, and all modules are taken to be unitary.

\section{Part I}

In what follows, $\mathrm{N}$ will be an ideal of $\mathrm{R}$, usually assumed to be contained in the Jacobson radical of $R$, here denoted Rad $R$. We say that idempotents modulo $N$ can be lifted provided for every element $u$ of $R$ such that $u^{2}-\overline{u \in N}$ there exists an idempotent $e^{2}=e \in R$ such that $e-u \in N$. In other words, if $\mathrm{u}$ is an idempotent modulo $\mathrm{N}$ then there shall exist an idempotent of $R$ to which it is congruent modulo $N$.

Canad. Math. Bull. vol. 8, no. 3, April 1965 
LEMMA 1. Assume that idempotents can be lifted modulc $N=\operatorname{Rad} R$. If $g$ is a given idempotent of $R$, and if $u$ is an idempotent modulo $N$ such that $u g$ and $g u \in N$, then there exists an idempotent $e$ of $R$ such that $e-u \in N$ and eg $=$ ge $=0$.

Proof. By assumption we may find $f^{2}=f \in R$ such that $f-u \in N$. It follows that $f g$ and gf $\epsilon$. In particular, $1-f g$ is a unit of $R$, and we may put

$$
f^{\prime}=(1-f g)^{-1} f(1-f g) .
$$

Clearly $f^{\prime}$ is an idempotent and $f^{\prime} g=0$. Multiplying by $1-f g$ on the left, we see that $f^{\prime}-f \in N$.

Now put $e=f^{\prime}-g f^{\prime}$. Then clearly $g e=0=e g, e^{2}=e$, and $e \equiv(1-g) f \equiv f \equiv u$ modulo $N$.

A set of idempotents is said to be orthogonal if the product of any two of them is zero.

PROPOSITION 1. Assume that idempotents can be lifted modulo $N C \operatorname{Rad} R$. Then any countable orthogonal set of nonzero idempotents modulo $\mathrm{N}$ can be lifted to an orthogonal set of non-zero idempotents of $R$.

Proof. We are given $u_{1}, u_{2}, \ldots \in R$ such that $u_{i}^{2}=\overline{u_{i} \neq 0}$ and $u_{i} u_{j}=0$ for $i \neq j$. Suppose we have already lifted $u_{1}, u_{2}, \ldots, u_{k-1}$ to the orthogonal set of idempotents $e_{1}, e_{2}, \ldots, e_{k-1}$. Put $g=e_{1}+\ldots+e_{k-1}$, then surely $u_{k} g$ and $g u_{k} \in N$. By the lemma, we can find an idempotent $e_{k} \equiv u_{k}$ modulo $N$ which is orthogonal to $g$, and hence to $e_{1}, e_{2}, \ldots, e_{k-1}$. Finally $e_{i} \neq 0$ since $u_{i} \& N$.

This result appears in the book by Jacobson (Chapter III, $\S 8$, Proposition 5), but its validity there is restricted to the so-called "SBI rings". The exact definition of this term need not concern us here, sirce we propose to replace it by the apparently more general concept "rings in which idempotents can be lifted" in a number of other results as well. 
For completeness we include the following:

LEMMA 2. If $e$ and $f$ are idempotents of $R$ and if $u^{\prime} u$ - $e$ and $u u^{\prime}-f \in \operatorname{Rad} R$, then there exist elements $v$ and $v^{\prime}$ such that $v^{\prime} v=e$ and $v v^{\prime}=f$.

This is proved essentially like III, $\S 8$, Proposition 1 of Jacobson's book.

A non-zero idempotent is called primitive if it cannot be written as the sum of two orthogonal non-zero idempotents. It is easily seen that the idempotent $e \in R$ is primitive if and only if the ring eRe contains no idempotents other than 0 and $e$.

LEMMA 3. Assume that idempotents car be lifted modulo $N \subset \operatorname{Rad} R$. Then any primitive idempotent of $R$ remains primitive modulo $\mathrm{N}$.

Proof. Suppose $e$ is a primitive idempotent and $\mathrm{e}=\mathrm{u}+\mathrm{v}$, where $\mathrm{u}$ and $\mathrm{v}$ are orthogonal idempotents modulo N. Then $u$ is orthogonal modulo $N$ to 1 - e. By Lemma 1, $u$ may be lifted to an idempotent $f$ of $R$ which is orthogonal to 1-e, thus $f \in e$ Re. By primitivity of $e, f=0$ or $e$, hence $u \equiv 0$ or e modulo $N$. Thus $e$ is primitive modulo $N$.

We follow Bass in calling the ring $R$ semi-perfect if idempotents can be lifted modulo $\operatorname{Rad} R$ and $R / \operatorname{Rad} R$ is completely reducible (that is, artinian semi-simple).

PROPOSITION 2. Any semi-perfect ring contains a finite orthogonal set of primitive idempotents whose sum is 1 .

This is stated in Jacobson III, $\$ 9$, Theorem 4 for semiprimary SBI rings. The proof there is left as an exercise to the reader. Well, the same proof will yield the present result.

A ring $R$ is called local if $R / \operatorname{Rad} R$ is a division $r i n g$, or equivalently, if the non-units of $R$ form an ideal. (Jacobson calls such a ring "completely primary".)

LEMMA 4. If $\mathrm{e}$ is a primitive idempotent in a semiperfect ring $R$ then $e R e$ is a local ring. 
Proof. First let us observe that $\mathrm{e}$ is the unity element of eRe. Furthermore, if $\operatorname{Rad} R=N$, then $\operatorname{Rad}(e \operatorname{Re})=e \mathrm{Ne}$ $=$ eRe $\cap N$ (see Jacobson III, §7, Proposition 1).

Next consider any element $u \in$ eRe. Since $R / N$ is a regular ring (in the sense of Von Neumann), there exists $u^{\prime} \in R$ such that $u u^{\prime} u \equiv u$ modulo $N$. Since we can always replace $u^{\prime}$ by eu'e in this, we may as well assume that $u^{\prime} \epsilon$ eRe also.

Now uu' is an idempotent modulo $N$ orthogonal to 1 -e. By Lemma 1, we can lift it to an idempotent $f$ of $R$ orthogonal to 1 - e. Thus $f \in$ eRe, and $f$ and $e-f$ are two orthogonal idempotents. Since $\mathrm{e}$ is primitive, one of them must be 0 .

We shall now assume that $u \neq 0$ modulo $N$. Since $f u \equiv u u^{\prime} u \equiv u$ modulo $N$, it follows that $f \neq 0$. Therefore $f=e$, and so $u^{\prime} \equiv e$ modulo $N$. Similarly $u^{\prime} u \equiv e$ modulo $N$, hence $\mathrm{u}$ is a unit of eRe modulo $\mathrm{N} \cap \mathrm{e} \operatorname{Re}=\operatorname{Rad}(e \operatorname{Re})$. Thus eRe is a local ring.

PROPOSITION 3. If $R$ is semi-perfect and $R /$ Rad $R$ is simple, then $R$ is isomorphic to the ring of all endomorphisn of a finitely generated free module over a local ring.

This may be proved as in Jacobson, III, $\S 8$, Theorem 1 or as Proposition 6 in Part II below.

\section{Part II}

To set the stage for our main result, we introduce some notation and review some known facts.

Let $L$ be an $R$-submodule of the R-module $M$, and suppose that $L$ has non-zero intersection with every non-zero submodule of $M$. Then $L$ is called a large submodule of $M$, or $M$ is called an essential extension of $L$.

Given any $R$-module $M_{R}$. As Eckmann and Schopf have shown, $M_{R}$ has exactly one (up to isomorphism) essential extension which is also injective. It is called the injective hull 
of $M_{R}$, and we denote it by $I_{R}=I\left(M_{R}\right)$. It is both a maximal essential extension and a minimal injective extension of $M_{R}$.

We write $\mathrm{H}=\mathrm{H}\left(\mathrm{M}_{\mathrm{R}}\right)=\operatorname{Hom}_{\mathrm{R}}(\mathrm{I}, \mathrm{I})$. Then $\mathrm{I}$ becomes a bimodule $H_{R}$. We also write $Q\left(M_{R}\right)=\operatorname{Hom}_{H}(I, I)$. The following results are known.

(A) (Utumi 1959) Rad H consists of all those elements of $\mathrm{H}$ which annihilate a large submodule of $I_{R}$ or, what comes to the same, of $M_{R}$.

(B) (Johnson 1951) $\mathrm{H} / \mathrm{Rad} \mathrm{H}$ is a regular ring, called the extended centralizer of $R$ over $M$.

(C) (Lambek 1963) $Q=Q\left(R_{R}\right)$ is a faithful extension of $R$. It coincides with Utumi's (maximal) ring of right quotients of $R$. It is also the largest of Gabriel's rings of right quotients which faithfully extend $R$ (as expounded in the exercises of Bourbaki XXVII).

No use of $Q\left(M_{R}\right)$ for $M \neq R$ will be made here.

PROPOSITION 4. In $\mathrm{H}=\operatorname{Hom}_{\mathrm{R}}(\mathrm{I}, \mathrm{I})$ idempotents modulo Rad $\mathrm{H}$ can be lifted.

Both Utumi and Chase have mentioned to me in conversation that they have proved this result. A proof attributed to the latter appears in the notes by Faith. The following proof was obtained independently and differs from that by Chase.

Proof. Let $u \in H, u^{2}-u \in \operatorname{Rad} H$. Then there exists a large submodule $L$ of $I_{R}$ such that $\left(u^{2}-u\right) L=0$. The injective hull of $\mathrm{uL}$, being its minimal injective extension, can be embedded in the injective module $I_{R}$, hence it has the form eI, where $e^{2}=e \in H$. Since $e$ induces the identity mapping on $u L$, we have $(e u-u) L=0$, hence eu $\equiv u$ modulo Rad $H$. 
Next, put $f=e+e u(1-e)$. Then $e f=f, f e=e$ and $f^{2}=f$. Let $L^{\prime}=(1-e) I+u L$. A routine computation show that $L^{\prime}$ is a large submodule of $I_{R}$ and that $(f-e u) L^{\prime}=0$. Therefore $f \equiv e u=u$ modulo $\operatorname{Rad} H$, as required.

We follow Goldie in calling the module $\dot{M}_{R}$ finitedimensional if there do not exist infinitely many non-zero submodules whose sum is direct. Clearly, all noetherian and all artinian modules are finite-dimensional.

PROPOSITION 5. Let $M_{R}$ be finite-dimensional. Then

(1) $I_{R}$ is the direct sum of a finite number of indecomposable injective modules,

(2) $\mathrm{H} / \mathrm{Rad} \mathrm{H}$ is completely reducible,

(3) $\mathrm{H}$ is semi-perfect.

Proof. (1) Consider any orthogonal set $E$ of non-zero idempotents of $H$. Then $\Sigma_{e \in E}$ eI $\frown M$ is a direct sum of non-zero submodules of $M_{R}$. By assumption, $E$ must be finite. One applies this principle twice. First, one shows that, for any non-zero idempotent $e$ of $\mathrm{H}$, eHe contains a primitive idempotent. (If $\mathrm{e}$ is not primitive, eHe contains an idempotent $\mathrm{e}_{1} \neq \mathrm{e}, 0$. If $\mathrm{e}_{1}$ is not primitive, $\mathrm{e}_{1} \mathrm{He}{ }_{1}$ contains an idempotent $e_{2} \neq e_{1}, 0$, and so on. The idempotents $e-e_{1}, e_{1}-e_{2}, \ldots$ form an orthogonal set, which must be finite.) Secondly, to show that there exists a maximal orthogonal set $\mathrm{e}_{1}, \mathrm{e}_{2}, \ldots, \mathrm{e}_{\mathrm{n}}$ of primitive idempotents in $H$, let $e$ be their sum and suppose that $\mathrm{e} \neq 1$, then $(1-\mathrm{e}) \mathrm{R}(1-\mathrm{e})$ would contain a primitive idempotent orthogonal to $e$, hence to all $e_{i}$, a contradiction.

(2) By the above, there is a finite orthogonal set of primitive idempotents whose sum is 1. By Lemma 3, the same is true modulo Rad $H$, hence $\bar{H}=H /$ Rad $H$ is a direct sum of indecomposable right ideals. Since $\bar{H}$ is regular, these are minimal right ideals. Therefore $\bar{H}$ is completely reducible. 
(3) This follows immediately from (2) and Proposition 4.

COROLLARY. Let $M_{R}$ be finite-dimensional and suppose the indecomposable components of $I_{R}$ are all isomorphic. Then $\mathrm{H} / \mathrm{Rad} \mathrm{H}$ and $\mathrm{H}$ are isomorphic to the rings of all endomorphisms of finitely generated free modules over a division ring and a local ring respectively.

The proofs use the classical Artin-Wedderburn Theorem and Proposition 3 respectively.

COMMENT. Proposition 5 is not new. (1) may easily be deduced from a theorem of Matlis, and (2) appears in the paper "Coeur d' un module" by Lesieur and Croisot (Theorem 2. 2).

PROPOSITION 6. Let $R_{R}$ be finite-dimensional and suppose the indecomposable components of its injective hull $I\left(R_{R}\right)$ are all isomorphic. Then Utumi's ring of right quotients $Q\left(R_{R}\right)$ is isomorphic to the ring of all endomorphisms of a finitely generated module over a local ring.

Proof. By (1) of Proposition 5, the unity element of $\mathrm{H}$ is the sum of primitive idempotents $\mathrm{e}=\mathrm{e}_{1}, \mathrm{e}_{2}, \ldots, \mathrm{e}_{\mathrm{n}}$. It is assumed that all $e_{k} I$ are isomorphic to eI. Hence there exist $u_{k}$ and $v_{k} \in H$ such that $v_{k} u_{k}=e_{k}$ and $u_{k} v_{k}=e$.

Now consider $Q=\operatorname{Hom}_{H}(I, I)$. By a well-known argument, this is isomorphic to $\operatorname{Hom}_{e H e}(e I, e I)$. Indeed, let $q \in Q$, and define $q^{\prime}:$ eI $\rightarrow$ I by $(e i) q^{\prime}=(e i) q=e(e i) q . \quad$ Compute $\mathrm{v}_{\mathrm{k}} \mathrm{eu}_{\mathrm{k}}=\mathrm{v}_{\mathrm{k}} \mathrm{u}_{\mathrm{k}} \mathrm{v}_{\mathrm{k}} \mathrm{u}_{\mathrm{k}}=\mathrm{e}_{\mathrm{k}}^{2}=\mathrm{e}_{\mathrm{k}}$. For any $\mathrm{i}=\mathrm{\Sigma}_{\mathrm{k}=1}^{\mathrm{n}} \mathrm{e}_{\mathrm{k}}^{\mathrm{i}}=$ $\sum_{k=1}^{n} v_{k} e_{k} i \in I$ we then have

$$
\text { iq }=\Sigma v_{k} e u_{k} i q=\Sigma v_{k}\left(e u_{k} i\right) q^{\prime}
$$

From this formula one readily infers that the correspondence $\mathrm{q} \rightarrow \mathrm{q}^{\prime}$ is an isomorphism. 
Next, observe that $\mathrm{eH}=\Sigma_{k-1}^{n}$ eHe ${ }_{k}=\Sigma_{k-1}^{n}$ eHeu ${ }_{k}$, since e $u_{k}=u_{k} e_{k}$ and $e_{k}=v_{k} e_{k} u_{k}$ Since the $e_{k}$ are orthogonal idempotents, one easily sees that this is a direct sum. Thus $\mathrm{eH}$ is a finitely generated free eHe-module. Now, if 1 denotes the unity element of $R, h \rightarrow h 1$ is an epimorphism of $\mathrm{H}^{\mathrm{H}}$ onto $H^{I}$ Hence $I=e H 1$, and so $e I$ is also a finitely generated eHemodule, although there is no longer any reason for it to be free.

Finally, eHe is a local ring by Lemma 4, and so our proof is complete.

It would be pleasant if we could replace the condition "all indecomposable components of $I\left(R_{R}\right)$ are isomorphic" by a neat internal characterization of $R$. This can be done for noetherian $R$ in view of a result by Lesieur and Croisot (see the very last italicized statement in their book). For completeness, we repeat it here in our own words.

PROPOSITION 7. Let $R$ be both right and left noetherian. Then the indecomposable components of $I\left(R_{R}\right)$ are all isomorphic if and only if $R_{R}$ is tertiary in the sense that for every ideal $A$ of $R$ the left annihilator $\{r \in R \mid r A=0\}$ is either 0 or large as a right ideal.

\section{Part III}

Johnson introduced the singular submodule $J\left(M_{R}\right)$ of a module $M_{R}$ as the set of all elements of $M$ which annihilate some large right ideal of $R$. This can also be defined as the intersection of $M$ with the radical of $H^{I}$, in view of $(A)$ above.

Rings for which $J\left(R_{R}\right)=0$ have been extensively studied by Johnson. They are called (right) neat in Bourbaki XXVII. It is well known and not difficult to show that for such rings also $\operatorname{Rad} H=0$. Indeed, let $J\left(R_{R}\right)=0$, and put $K=\{h \in H \mid h R=0\}$. Then $K I=(\operatorname{Rad} H) H 1 C(\operatorname{Rad} H) 1=J\left(I_{R}\right)=0$. Now it is known 
that $1 Q=\{i \in I \mid K i=0\}$ (see for instance Lambek 1963), hence $1 Q=I$. Therefore $H \cong Q \cong I=I / J\left(I_{R}\right) \cong H / R a d H$ is a regular ring and so $\mathrm{Rad} H=0$.

The following result is due to Johnson (see his paper of 1961, Theorem 4.3), but is here presented as a corollary to Proposition 5 or 6.

COROLLARY. Let $R_{R}$ be neat and finite dimensional. Then $\mathrm{Q} \cong \mathrm{H}$ is completely reducible.

If $R$ is moreover semi-prime, Goldie showed that $Q$ is the classical ring of right quotients of $R$, in the sense that every non-zero-divisor of $R$ is a unit in $Q$ and that every element of $Q$ has the form $\mathbf{r s}^{-1}$ with $r$ and $s \in R$.

It is not difficult to show that $Q\left(R_{R}\right)$ will be classical if every large right ideal of $R$ contains a unit of $Q$. The following crucial lemma goes back to Goldie, but its simple proof occurred to me upon reading another proof by Johnson and Wong, part of whose argument it still contains.

LEMMA 5. If $R$ is prime and $Q$ is the ring of all $n$ by $n$ matrices over a division ring then every large right ideal $L$ of $R$ contains a unit of $Q$.

Proof. Since $Q$ is left noetherian, we can find a $\in L$ so that $\mathrm{Qa}$ is maximal. Since $Q$ is regular, we can find $a^{\prime} \in Q$ such that $a a^{\prime} a=a$. Now $e=a a^{\prime}$ and $f=a^{\prime} a$ are idempotents; we shall see that they are both 1.

Consider any $b \in(1-e) Q \rightarrow L$. Then $e(a+b)=a$; hence $Q a: Q(a+b)$, with $a+b \in L$. Since $Q a$ was maximal with $a \in L$, it follows that $b \in Q a$. Thus $(1-\mathrm{e}) \mathrm{Q} \cap \mathrm{L}<\mathrm{Qa}$. Now $a(1-f)=0$, hence

$$
((1-e) Q \cap L)((1-f) Q \cap L)=0
$$

Since $R$ is prime, one of the factors is 0 . Since $L$ is large, either $e=1$ or $f=1$. Thus a has either a right inverse or a left inverse in $Q$. Being just a finite matrix with entries in a division ring it has both. 
It is not difficult to extend this result to semi-prime rings, using the fact that the annihilator ideals of a semi-prime ring form a boolean algebra, but we shall refrain from doing so here. Let us also point out that classical rings of quotients of noetherian rings which are not semi-prime have been studied by Feller and Swokowski and also by Talentyre.

The above proof of Lemma 5 is very similar to the proof of the corresponding result for semi-prime rings in the lecture notes by Faith. Nonetheless it seemed worth pointing out the simplification which arises in the prime case.

Let me take this opportunity to correct a statement made earlier. In my paper of $1963 \mathrm{I}$ asserted that in all known examples for which $Q$ is not classical, $R$ fails to satisfy the maximum condition for right ideals. Carl Faith has pointed out that the 1958 paper with Findlay does in fact exhibit a finite ring $R$ for which $Q \neq R$ (Example 9.3). The non-zero-divisors of $\mathrm{R}$ form a finite cancellation semi-group, which must be a group, and so $R$ is its own classical ring of right quotients.

\section{REFERENCES}

1. H. Bass, Finitistic homological dimension and a homological generalization of semi-primary rings, Trans. Amer. Math. Soc. 95 (1960), 466-488.

2. B. Eckmann and A. Schopf, Über Injektive Moduln, Archiv der Math. 4 (1953), 75-78.

3. C. Faith, Lectures on injective modules and quotient rings, Rutgers 1964. (Multilithed.)

4. E. H. Feller and E. W. Swokowski, On ring extensions for completely primary noncommutative rings, Trans. Amer. Math. Soc. 105 (1962), 251-263.

5. G. D. Findlay and J. Lambek, A generalized ring of quotients II, Can. Math. Bull. 1 (1958), 155-167. 
6. A. W. Goldie, The structure of prime rings under ascending chain conditions, Proc. London Math. Soc. 8 (1958), 589-608.

7. , Semi-prime rings with maximum condition, Proc. London Math. Soc. 10 (1960), 201-220.

8. $\overline{\text { (Multilithed.) }}$ , Rings with maximum condition, Yale 1961.

9. N. Jacobson, Structure of rings, Providence 1956.

10. R. E. Johnson, The extended centralizer of a ring over a module, Proc. Amer. Math. Soc. 2 (1951), 891-895.

11. , Structure theory of faithful rings, II. Restricted rings, Trans. Amer. Math. Soc. 84 (1957), 523-544. III. Irreducible rings, Proc. Amer. Math. Soc. $11(1960), 710-717$.

12. , Quotient rings of rings with zero singular ideal, Pac. J. Math. 11 (1961), 1385-1392.

13. R. E. Johnson and E. T. Wong, Quasi-injective modules and irreducible rings, J. London Math. Soc. 36 (1961), 260-268.

14. J. Lambek, On Utumi's ring of quotients, Can. J. Math. 15 (1963), 363-370.

15. L. Lesieur and R. Croisot, Algèbre noethérienne non commutative, Paris 1963.

16. , Coeur d'un module, Journal de Mathématique $\overline{42(1963), 367}-407$.

17. E. Matlis, Injective modules over noetherian rings, Pac. J. Math. 8 (1958), 511-528.

18. T.D. Talentyre, Quotient rings of rings with maximum condition on right ideals, J. London Math. Soc. 38 (1963), 439-450. 
19. Y. Utumi, On quotient rings, Osaka Math. J. 8 (1956), $1-18$.

20. , On a theorem on modular lattices, Proc. Japan Acad. 35 (1959), 16-21.

McGill University and

Summer Research Institute, Canadian Mathematical Congress 